\title{
Enhanced Obstetrics Training for Family Practice Residents: A Unique Collaborative Program
}

\author{
Jim Nuovo, $M D$, and Lloyd Smith, $M D, P b D$
}

Access to obstetric services in rural areas has been and continues to be a challenge. ${ }^{1-4}$ When available, much of the obstetric care in these areas has been provided by family physicians. ${ }^{1,5}$ Unfortunately, the number of family physicians who practice obstetrics has been in decline. In 1996 only $26 \%$ of active members of the American Academy of Family Physicians included obstetrics in their practice. ${ }^{6}$ Although rural family physicians are more likely than their urban counterparts to provide maternity care, less than one half perform routine deliveries. ${ }^{5}$

The obstetric services needed in rural areas include access to providers who can perform cesarean deliveries. ${ }^{7}$ Norris et $\mathrm{al}^{8}$ performed a survey of 41 rural hospitals in Washington State. They found that most of these hospitals depend on family physicians for this operative intervention. Furthermore, they found that a family physician's comfort in doing cesarean deliveries was closely related to their formal training during residency. Opportunities for advanced obstetric training have included the following: (1) an emphasis on obstetrics (including operative obstetrics) during residency training; (2) 1-year fellowship programs (typically community-based programs that emphasize skills for rural practice including obstetrics) ${ }^{9,10}$; (3) onthe-job training in the communities or in some other nonresidency setting ${ }^{8}$; (4) rural training tracks with a strong emphasis on obstetrics ${ }^{11}$; and (5) combined family practice-obstetrics programs. $^{12}$

Although the importance of having family physician educators to model advanced obstetric skills is critical, it is also important to improve educational ties between departments of family practice and obstetrics and gynecology. As stated by Caudle et al, "It is time for academic medical centers to

Submitted, revised, 10 November 1998.

From the Department of Family and Community Medicine (JN), and the Department of Obstetrics and Gynecology (LS), University of California, Davis. Address reprint requests to Jim Nuovo, MD, Department of Family and Community Medicine, University of California, Davis, 4860 Y St, Sacramento, CA 95817. adjust their philosophy and place a greater priority on advanced obstetric training for family physicians. Motivated obstetric faculty and family practice faculty are both essential to such an effort." Recently, there has been interest in improving collaboration between the specialties of family practice and obstetrics and gynecology. This interest has resulted in a consensus publication of recommended core educational guidelines by the American Academy of Family Practice and the American College of Obstetricians and Gynecologists. ${ }^{13}$

The purpose of this article is to describe an example of collaboration between academic departments of family practice and obstetrics and gynecology at the University of California, Davis, which lead to the development of a 4-year track for family practice residents specifically designed for training in advanced obstetrics.

\section{Program Development}

The faculty of the Departments of Family and Community Medicine and Obstetrics and Gynecology reached consensus on both the need for and importance of a combined family practice-obstetrics program. This consensus was reached through presentations at faculty meetings. Key points of discussion included an understanding of the need for obstetric services in rural areas, the previous success of the Department of Family Practice in placing graduates in rural areas, the results of surveys of graduates of the family practice program showing a need for advanced obstetric skills, and recently a developed curriculum to provide primary care training for obstetrics-gynecology residents in the family practice department. From these discussions the faculty agreed that the primary objective was to provide an opportunity for residents to complete family practice training with enhanced obstetric skills (including operative obstetrics) while maintaining the other skills expected of our family practice graduates in the standard track.

The proposal was presented to the Graduate Medical Education Advisory Committee (GMEAC) 
and approved. The timing was believed to be opportune, as the committee had ongoing efforts to enhance the number of primary care physicians through redistribution of specialty slots. Our presentation to the GMEAC included a request to reduce the number of residents in the obstetricsgynecology program by 1 per year and to increase the number of residents in the family practice program by 2 per year.

The curriculum was developed using as a model the guidelines published by the American Board of Family Practice (ABFP) and American Board of Psychiatry and Neurology for the combined family practice-psychiatry program. The most notable difference between these two programs is that dual board certification in family practice and obstetrics-gynecology is not a goal of this program. We believed that the additional requirement of operative gynecology training would be counterproductive to the primary objective, namely, achieving advanced obstetric skills. The specific curriculum was developed and submitted to the ABFP and approved. The residency training program directors from each department were designated as codirectors for the collaborative program. A steering committee with faculty from each department was established to meet quarterly to review resident performance, share evaluations, and coordinate and evaluate the curriculum. A process for interview and selection of applicants for the combined program was developed. The entire process from vision to implementation took 2 years.

\section{The Collaborative Program}

The curriculum for the collaborative program is outlined in Table 1. Salient features of the curriculum are as follows:

1. The program is of 4 years' duration.

2. The resident meets all of the Residency Review Committee requirements for family practice training during this time.

3. Obstetric training is increased to 18 blocks (one block equals a 4-week rotation).

4. The content of the obstetric training for the residents in the combined program is identical to that for obstetric-gynecology residents.

5. Gynecology training for the residents is outpatient based.
Table 1. Curriculum for the University of California, Davis, Collaborative Family Practice-Obstetrics Program.

\begin{tabular}{lcc}
\hline Rotation & $\begin{array}{c}\text { Collaborative Track } \\
\text { Blocks }\end{array}$ & $\begin{array}{c}\text { Regular Track } \\
\text { Blocks }\end{array}$ \\
\hline Cardiology & 0.5 & 0.5 \\
$\begin{array}{l}\text { Community } \\
\text { medicine }\end{array}$ & 1 & 1 \\
Electives & 3 & 6 \\
Emergency medicine & 1 & 1 \\
Ear, nose, and throat & 0.5 & 0.5 \\
Dermatology & 1 & 1 \\
Didactics & 2 & 2 \\
Gynecology & 4 & 1 \\
Medicine & 8 & 9 \\
Obstetrics & 18 & 3 \\
Ophthalmiology & 0.5 & 0.5 \\
Orthopedics & 1 & 1 \\
Procedure clinic & 1 & 2 \\
Pediatrics & 4 & 5 \\
Surgery & 2 & 2 \\
Urology & 0.5 & 0.5 \\
Total & 48 & 36 \\
\hline
\end{tabular}

6. The resident is required to attend continuity clinics in the family practice center during all 4 years, specifically, 1 half-day per week in the first year, 2 half-days per week in the second year, and 3 half-days per week in the third and fourth years.

The program will ultimately be comprised of 4 residents each year. Necessary changes in the rotation, call, and clinic schedules were made in each program to accommodate the combined program and to continue to meet the respective Residency Review Committee requirements.

\section{Significant Issues \\ Resident Stress}

In combined programs residents are challenged to develop expertise in two specialties. Trainees might experience increased stress, particularly when switching rotations from one specialty to the other (so-called switch stress). Residents in our collaborative program might experience similar problems with identity. Without available role models or a peer group, there is an increased chance of isolation. In addition, residents in combined programs risk becoming lost between the two departments as a result of their limited contact with peers who have 
Table 2. Specific Educational Experiences in Obstetrics.

1. Full range of obstetrics, including high-risk obstetrics and medical and surgical complications of pregnancy

2. Genetics, including genetic amniocentesis and patient counseling

3. Operative vaginal deliveries, including obstetric forceps or vacuum extractor

4. Vaginal breech and vaginal multifetal deliveries

5. Vaginal births after cesarean delivery

6. Obstetric anesthesia. Residents must learn the principles of general and conduction anesthesia, together with the management and the complications of these techniques

7. Management of critically ill patients

8. Immediate care of the newborn. Every resident must have experience in resuscitating the human newborn, including tracheal intubation. The principles of general neonatal complications must be learned as well

9. Full range of common obstetric diagnostic procedures, including sonography and other relevant imaging techniques

10. Emotional and psychologic impact of pregnancy upon a woman and her family in a variety of circumstances

11. Counseling women regarding nutrition, exercise, health maintenance, high-risk behaviors, and preparation for pregnancy and childbirth

12. Obstetric pathology

similar interests and career plans. Easy access to a faculty coordinator who acts as a liaison between the two departments contributes to the resident's sense of unity. ${ }^{14-16}$

\section{Clarifying Educational Goals}

It is important that residents have clear educational goals for the collaborative program. Ultimately, the goal of this program is to give trainees an opportunity to develop skills necessary to provide the full spectrum of family practice and obstetric services to their patients. We believe that the optimal way to clarify the educational goals is to maintain the complete training goals described in the family practice Residency Review Committee special requirements and the specific educational objectives in obstetrics described in the obstetrics-gynecology Residency Review Committee special requirements. These objectives are listed in Table $2 .{ }^{17}$

\section{Ensuring the Strength and Viability of Each Independent Program}

Combined programs put additional stress on the service and educational components of the regular track residents of each program by taking away resources for rotation, call, and clinic schedules.
These components are particularly stressed when allocations for collaborative positions are taken away from the current pool of residents. It takes extra effort to coordinate these changes so that the impact is minimized. ${ }^{14-16}$ In our experience, it was necessary to restructure some of the regular track rotations to avoid overburdening these residents with service coverage.

\section{Integrated Training}

As noted, coordinating the many components of the curriculum, such as continuity clinic, conference, rotation, and call schedules, is challenging. A collaborative program inevitably means residents are sometimes scheduled for two events at the same time. As residents in a collaborative program are trying to master the knowledge and skills in two specialties, it is important to consider educational opportunities that might integrate training for these residents.

\section{Acceptance of Collaborative Residents}

Problems encountered in obtaining obstetric privileges for family practice graduates are well-documented. ${ }^{18}$ As with other programs to provide advanced obstetrics training to family physicians, there will be hospitals and providers who will not accept this training as equal to that provided to an obstetrics-gynecology resident. Our curriculum was specifically structured to challenge this argument in that the graduates of our collaborative program should have knowledge and skills in obstetrics that are identical to those of obstetricsgynecology residents. We believe it is important to reiterate the American College of Obstetrics and Gynecology national guidelines on this issue, namely, privileges should be based on experience and training rather than specific membership or board certification.

\section{Ensuring Placement in Areas of Need}

The development of a collaborative family practice-obstetrics program by itself will not ensure placement of graduates in areas of need. Family practice programs that have been successful in graduating residents who practice in underserved areas have found it is important not only to recruit applicants who are likely to select such practice opportunities but also to provide training experiences during residency that allow the residents the chance to work at such sites. 
Comparing Options for Acbieving Advanced Training in Obstetrics

As noted, there are other opportunities for family physicians to obtain advanced training in obstetrics, and no research evidence indicates a preference for any one of these models. In our opinion, there is no reason to imply that any one model is best. Certainly individual styles of learning will influence which type of program a particular candidate selects for this training. It will be important, however, for educators to track carefully the specific training experiences in each model as well as the impact on the practice style of its graduates. These data will form the basis for useful comparisons in the future.

\section{Additional Collaborative Benefits}

We believe there are substantial advantages to collaboration between academic departments that go beyond graduation of residents in a collaborative program. This opportunity represents one example of a fundamental change needed in graduate medical education, ie, enhanced relationships and expanded teamwork requiring new beliefs and normative elements in the medical culture. ${ }^{19}$ Collaborative training adds the potential of other enhancements. In our situation, we are working on developing a co-located primary care practice, a multidisciplinary women's health center, and increased sharing of faculty preceptors in the outpatient setting.

\section{Conclusion}

There has been a growing interest in collaborative training programs at academic health centers. We have described a summary of one such program in family practice and obstetrics at the University of California, Davis. We believe that the graduates of this program will be competent in all operative procedures in obstetrics while still being able to provide the full services expected of a family physician. Although there are unique challenges to the development of a combined program, the benefits for interested residents and for the departments of family practice and obstetrics-gynecology make this endeavor a valuable opportunity for collaboration. We believe that in the end such efforts will work to serve at-need populations.
References

1. Caudle MR, Clapp M, Stockton D, Neutens J. Advanced obstetrical training for family physicians: the ${ }_{0}^{\circ}$ future hope for rural obstetrical care. J Fam Pract $\frac{\mathscr{O}}{2}$ 1995;41:123-5.

2. Deprez RD, Agger MS, McQuinn LB. Access to physicians, obstetric care use, and adequacy of pre- 0 natal care for Medicaid patients in Maine: 19851989. Obstet Gynecol 1996;88:443-50.

3. Nesbitt TS, Connell FA, Hart LG, Rosenblatt RA. Access to obstetric care in rural areas: effect on birth outcomes. Am J Public Health 1990;80:814-8.

4. Nesbitt TS, Larson EH, Rosenblatt RA, Hart LG. Access to maternity care in rural Washington: its effect on neonatal outcomes and resource use. Am J $\vec{\rho}$ Public Health 1997;87:85-90.

5. Pathman D, Tropman S. Obstetrical practice among $\vec{N}$ new rural family physicians. J Fam Pract 1995;40: $\frac{\sqrt{0}}{\vec{N}}$ $457-64$

6. Facts about family practice. Kansas City, Mo: Amer- $\frac{\vec{P}}{N}$ ican Academy of Family Physicians, 1996.

7. Deutchman $M$. Who ever heard of family physicians $\overrightarrow{0}$ performing cesarean sections? J Fam Pract 1996;43: $449-53$.

8. Norris TE, Reese JW, Pirani MJ, Rosenblatt RA. $\overrightarrow{\text { D }}$ Are rural family physicians comfortable performing $\frac{D}{2}$ cesarean sections? J Fam Pract 1996;43:455-60.

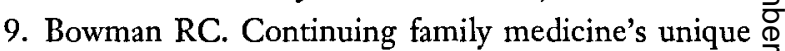
contribution to rural health care. Am Fam Physician $\vec{\bullet}$ 1996;54:471-4, 479, 483.

10. Norris TE, Acosta DA. A fellowship in rural family medicine: program development and outcomes. Fam Med 1997;29:414-20.

11. Rosenthal TC, McGuigan MH, Osborne J, Holden

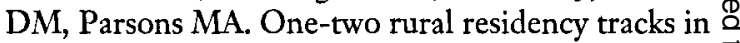
family practice: are they getting the job done? Fam $\overrightarrow{\overrightarrow{0}}$ Med 1998;30:90-3.

12. Frieden J. Joint Ob. Gyn.-FP residency formed. Fam Pract News 1997;27:1.

13. AAFP, ACOG reach consensus. Agreement boosts FP/OB interaction. FP Rep 1998;4:1.

14. Schubiner H, Schuster B, Moncrease A, Mosca C. The perspectives of current trainees in combined internal medicine-pediatrics. Results of a national survey. Am J Dis Child 1993;147:885-9.

15. Greganti MA, Schuster BL. Two combined resi- مै dency programs in internal medicine and pediatrics. $\frac{7}{2}$ J Med Educ 1986;61:883-92.

16. Lee MW. Weighing the benefits of combined resi- N $^{-}$ dency programs. JAMA 1991;266:1867.

17. Graduate medical education directory 1997-1998. Chicago: American Medical Association, 1997-1998.

18. Kahn NB Jr, Schmittling G. Obstetric privileges for $\stackrel{?}{?}$ family physicians: a national study. J Am Board Fam 꿍 Pract 1995;8:120-7.

19. Greenlick MR. Educating physicians for the twenty- $\frac{\Omega}{\mathbb{D}}$ first century. Acad Med 1995;70:179-85. 